\title{
Postsynaptic FMRP Promotes the Pruning of Cell-to-Cell Connections among Pyramidal Neurons in the L5A Neocortical Network
}

\author{
Ankur B. Patel, Kristofer W. Loerwald, Kimberly M. Huber, and Jay R. Gibson \\ University of Texas Southwestern Medical Center, Department of Neuroscience, Dallas, Texas 75390-9111
}

\begin{abstract}
Pruning of structural synapses occurs with development and learning. A deficit in pruning of cortical excitatory synapses and the resulting hyperconnectivity is hypothesized to underlie the etiology of fragile X syndrome (FXS) and related autistic disorders. However, clear evidence for pruning in neocortex and its impairment in FXS remains elusive. Using simultaneous recordings of pyramidal neurons in the layer 5A neocortical network of the wild-type (WT) mouse to observe cell-to-cell connections in isolation, we demonstrate here a specific form of "connection pruning." Connection frequency among pyramidal neurons decreases between the third and fifth postnatal weeks, indicating a period of connection pruning. Over the same interval in the FXS model mouse, the Fmrl knock-out (KO), connection frequency does not decrease. Therefore, connection frequency in the fifth week is higher in the Fmr1 KO compared with WT, indicating a state of hyperconnectivity. These alterations are due to postsynaptic deletion of Fmr1. At early ages (2 weeks), postsynaptic Fmr1 promoted the maturation of cell-to-cell connections, but not their number. These findings indicate that impaired connection pruning at later ages, and not an excess of connection formation, underlies the hyperconnectivity in the Fmr1 KO mouse. FMRP did not appear to regulate synapses individually, but instead regulated cell-to-cell connectivity in which groups of synapses mediating a single cell-to-cell connection are uniformly removed, retained, and matured. Although we do not link connection pruning directly to the pruning of structurally defined synapses, this study nevertheless provides an important model system for studying altered pruning in FXS.
\end{abstract}

Key words: circuit; cortex; development; electrophysiology; Fmr1; FMRP

\section{Introduction}

Circuit development involves a dynamic process of cooccurring synaptic formation and elimination. Synapse formation predominates during early postnatal stages, whereas, at later stages, elimination may predominate in some cortical pathways, resulting in a net "pruning" of synapses (Rakic et al., 1986; Holtmaat and Svoboda, 2009). Structural correlates of glutamatergic synapses, such as spines and synaptic markers, have been used to examine pruning during development, but these measurements have shortcomings. First, the relationship between structure and function of synapses is unclear. Second, multiple synaptic pathways are grouped together in this analysis, obscuring pruning processes and their mechanisms occurring in single pathways. Third, there is no way to distinguish between pruning of synapses and pruning of cell-to-cell connections. Functional measurements of pruning in a single synaptic pathway, such as that done in the

\footnotetext{
Received July 9, 2013; revised Jan. 9, 2014; accepted Jan. 29, 2014.

Author contributions: A.B.P., K.M.H., and J.R.G. designed research; A.B.P. and K.W.L. performed research; A.B.P., K.W.L., and J.R.G. analyzed data; A.B.P., K.M.H., and J.R.G. wrote the paper.

This work was supported by the National Institutes of Health (Grant HD056370 and linked ARRA administrative supplement to J.R.G., Grant HD052731 to K.M.H., and Training Grant 1T32NS069562 to A.B.P.) and Autism Speaks (K.M.H.). We thank Nicole Cabalo for technical assistance with mice.

Correspondence should be addressed to Jay R. Gibson, University of Texas, Southwestern Medical Center, Department of Neuroscience, Box 9111, Dallas, TX 75390-9111. E-mail: Jay.Gibson@UTSouthwestern.edu.

DOI:10.1523/JNEUROSCI.2921-13.2014

Copyright $\odot 2014$ the authors $\quad 0270-6474 / 14 / 343413-06 \$ 15.00 / 0$
}

cerebellum and lateral geniculate (Kano and Hashimoto, 2009), would circumvent these shortcomings.

Suppressed transcription of FMR1 and loss of its product, FMRP, is the main cause of fragile X syndrome (FXS), and a key hypothesis is that cognitive impairments in FXS are caused by the disruption of glutamatergic synaptic pruning in cortical structures and resulting circuit hyperconnectivity (Bagni and Greenough, 2005). This is consistent with a number of observations: (1) spine counts are higher in FXS patients and the Fmrl knockout (KO) mouse (Bagni and Greenough, 2005), (2) postsynaptic FMRP negatively regulates excitatory synapse number in cell culture (Pfeiffer and Huber, 2007), and (3) axonal overgrowth occurs with loss of FMRP (Bureau et al., 2008; Tessier and Broadie, 2008).

As measured with functional methods, neighboring layer 5 (L5) pyramidal neurons in prefrontal cortical slices of the Fmrl KO have a 1.7-fold higher connection frequency with each other (Testa-Silva et al., 2012). This hyperconnectivity was only observed during the third postnatal week. Although these results are consistent with a pruning deficit, no demonstration of pruning in the wild-type (WT) circuit was made, so no conclusions involving pruning were possible. Interestingly, there was no change in response amplitude in the cell-to-cell connections, suggesting that FMRP specifically regulates connectivity, not the strength of connections.

One alternative interpretation for much of the Fmrl KO data above is that there is an early period of excessive connection 

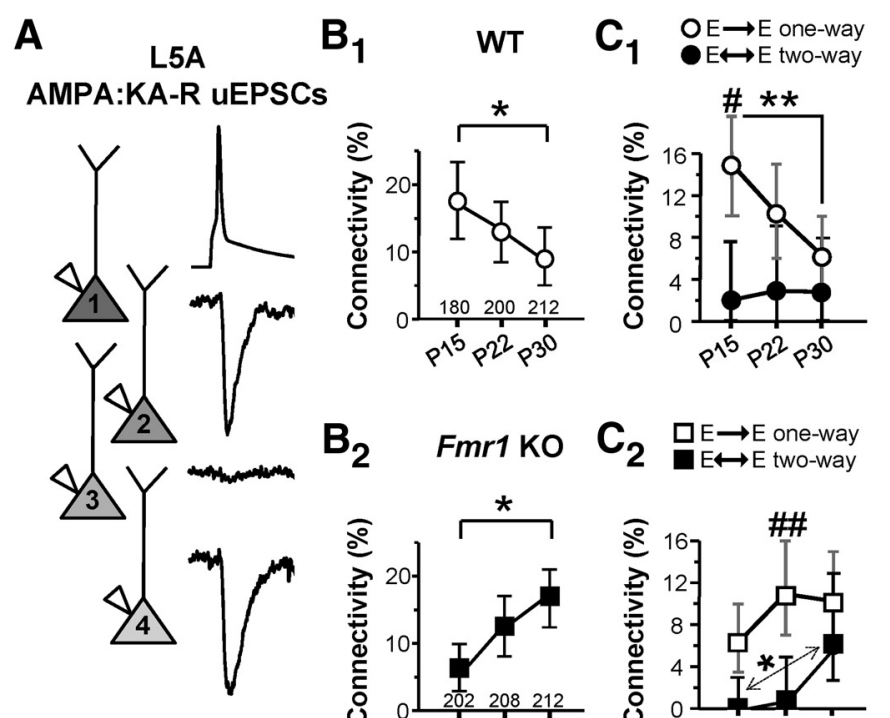

$D_{1}$
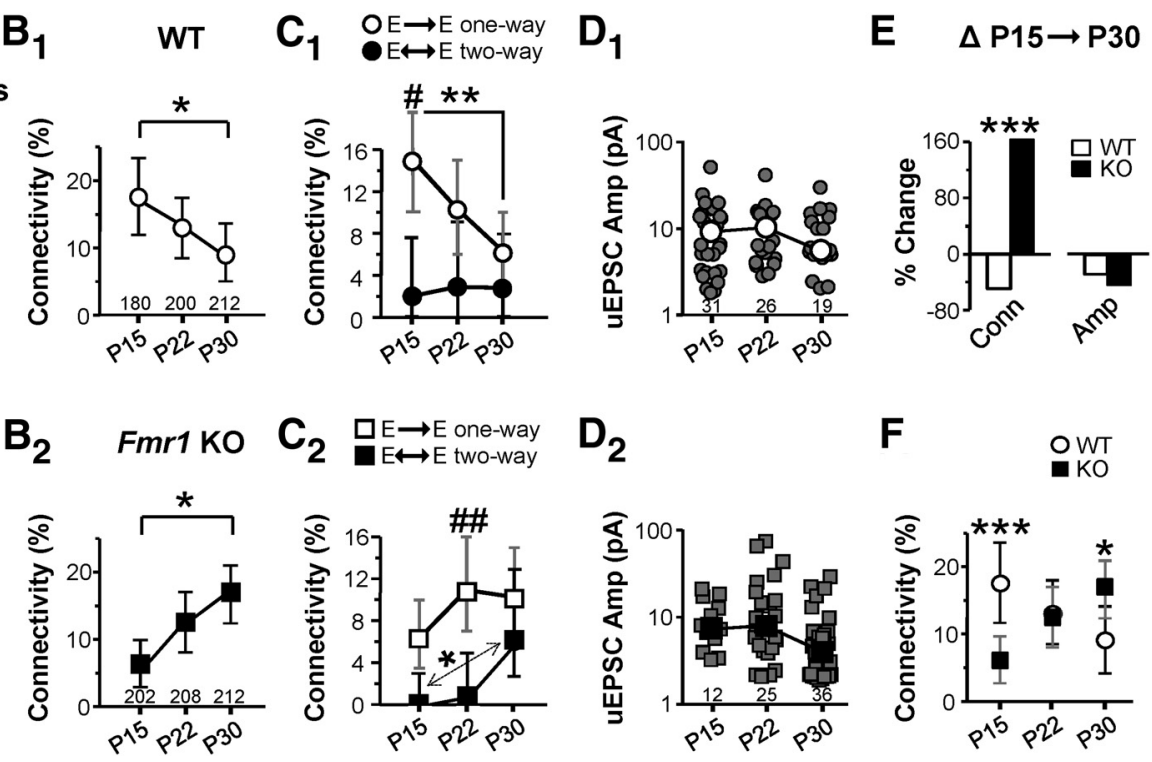

Figure 1. Loss of Fmr 1 disrupts the normal development of cell-to-cell connectivity mediated by AMPA:KA-Rs in L5a. $\boldsymbol{A}$, Example traces from pre- $(\boldsymbol{A} \mathbf{1})$ and postsynaptic neurons (A2, $\mathbf{A 3}, \boldsymbol{A 4})$ demonstrating two connections of a possible three as observed by average uEPSCS. B-D, Connectivity frequency $(\boldsymbol{B} 1, \boldsymbol{B} 2)$, frequency of connections being part of a "one-way" or "two-way" connection (C1, (2), and uEPSC amplitude (D1, D2) are plotted for WT (1) and Fmr $\mathrm{KO}$ (2) slices. Numbers in plots are total number of possible connections (B) and sample number (D). In $\boldsymbol{C}$, \#'s and *'s apply to one-vs two-way and P15 vs P30 comparisons, respectively. In D, medians are indicated. $\boldsymbol{E}$, Percent change in connectivity (Conn) and median amplitude (Amp) in the P15 $\rightarrow$ P30 interval. $\boldsymbol{F}$, Replot of $\boldsymbol{B}$ for comparison between genotypes. ${ }^{*} p<0.05 ;{ }^{* *} p<0.01 ;{ }^{* * *} p<0.001$ for all figures. Error bars in $\boldsymbol{B}, \boldsymbol{C}$, and $\boldsymbol{F}$ indicate $95 \%$ confidence interval.

formation and that the later pruning period is normal. Therefore, the developmental processes that lead to hyperconnectivity and excess synapses in vivo in the Fmrl KO remain unclear. Equally unclear is whether presynaptic or postsynaptic FMRP regulates cell-to-cell connectivity in vivo or if hyperconnectivity in the Fmr1 KO is even due to a cell-autonomous and direct effect of Fmr1. Only cell culture evidence exists and both a presynaptic and postsynaptic role for FMRP have been reported at hippocampal excitatory synapses (Hanson and Madison, 2007; Pfeiffer and Huber, 2007).

Using functional measurements in an isolated cortical pathway that normally undergoes "connection pruning" during development, we report here that pruning is specifically impaired with postsynaptic Fmr1 deletion.

\section{Materials and Methods}

Mice. Fmr1 KO mice (Bakker, 1994) and X-linked GFP mice (Hadjantonakis et al., 2001) were bred into the C57BL6 background for at least six generations. GFP/Fmr1 mosaic females were made by breeding X-linked GFP males with Fmr1 KO females, and no effects based on GFP expression are observed (Hanson and Madison, 2007; Patel et al., 2013). For all Fmr1 KO experiments, we used males because this makes littermate comparisons of KO versus WT more feasible. Three factors suggest that that the use of different sexes did not confound the results (for more details, see Patel et al., 2013): (1) most behavioral phenotypes appear similar in male and female Fmr1 KO mice; (2) females were, for the most part, too young to be in the estrus cycle, a regulator of neuronal properties; and (3) synaptic effects with Fmrl deletion in a previous study and in the present study were similar in both the mosaic and Fmrl KO (Patel et al., 2013).

Electrophysiology. Mice (P14-P32) were anesthetized with Euthasol and the brains removed following protocols approved by the University of Texas Southwestern Medical Center. Ages indicated in figures are accurate to \pm 2 . Preparation of thalamocortical slices, recording equipment, standard ACSF, and the K-gluconate pipette solution have been described previously (Patel et al., 2013). Simultaneous whole-cell recordings of up to four neurons ( $<35 \mu \mathrm{m}$ intersomal distance), which examined up to 12 possible one-way unitary connections, were performed in layer $5 \mathrm{~A}$ of somatosensory cortex at $21^{\circ} \mathrm{C}$. Miniature EPSCs were recorded in TTX $(1 \mu \mathrm{M})$ and picrotoxin $(25 \mu \mathrm{M})$.

L5A pyramidal neuron identity. L5A pyramidal neurons are almost all intratelencephalic projecting neurons that are a mix of intercortical and striatal projecting subtypes (Shepherd, 2013). It is very unlikely that large, pyramidal tract projecting neurons were included in our analysis because we avoided large somas, they are rare in L5A, and we excluded seven of 843 neurons based on their ability to evoke high-frequency bursts (a characteristic of pyramidal tract neurons). High-frequency bursts were defined at the minimum current step that evoked action potentials and had a first interspike interval of $<35 \mathrm{~ms}$ and an adaptation ratio (first divided by last interspike interval) $<0.8$. Inhibitory neurons were excluded based on firing properties and their ability to elicit a unitary IPSC (uIPSC).

AMPA and/or kainate receptor-mediated unitary EPSCs. Four presynaptic action potentials at $50 \mathrm{~ms}$ intervals were applied once every $10 \mathrm{~s}$ and measured at a holding potential of $-65 \mathrm{mV}$. Average unitary EPSC (uEPSC) amplitude was always determined from the first uEPSC in the train (uEPSC1) and was obtained from 20 trials (both successes and failures). Under these conditions, currents mediated by both AMPA-Rs and kainate receptors (KA-Rs) can be observed. Because of the ages examined and the fast time course of the uEPSCs, KA-Rs likely contributed very little (Contractor et al., 2011). A "connection" refers to a one-way, cell-to-cell connection and was detected when the average uEPSC amplitude was $>1.8 \mathrm{pA}$. Connectivity frequency was the percentage of detected connections out of all possible connections examined. An experiment was analyzed if the postsynaptic recording series resistance was $<25 \mathrm{M} \Omega$. In Figure $1 C$, a "one-way" connection meant that the postsynaptic neuron did not reciprocally project back to the presynaptic neuron. A "twoway" connection meant that the postsynaptic neuron did reciprocally project back to the presynaptic neuron. Short-term plasticity was obtained by calculating the amplitude ratio of both uEPSC2/uEPSC1 and average(uEPSC3,4)/uEPSC1. The coefficient of variation $(\mathrm{CV})$, risetime, and decay time constant were applied to uEPSC1 (average amplitude $>5 \mathrm{pA}$ ), as described previously (Patel et al., 2013).

NMDAR-mediated uEPSCs. Single presynaptic action potentials were applied once every $10 \mathrm{~s}$. First, uEPSCs mediated by AMPA:KA-Rs were collected. Then, DNQX $(20 \mu \mathrm{M})$, glycine $(20 \mu \mathrm{M})$, and $4 / 0 \mathrm{mM} \mathrm{Ca}^{2+} /$ 

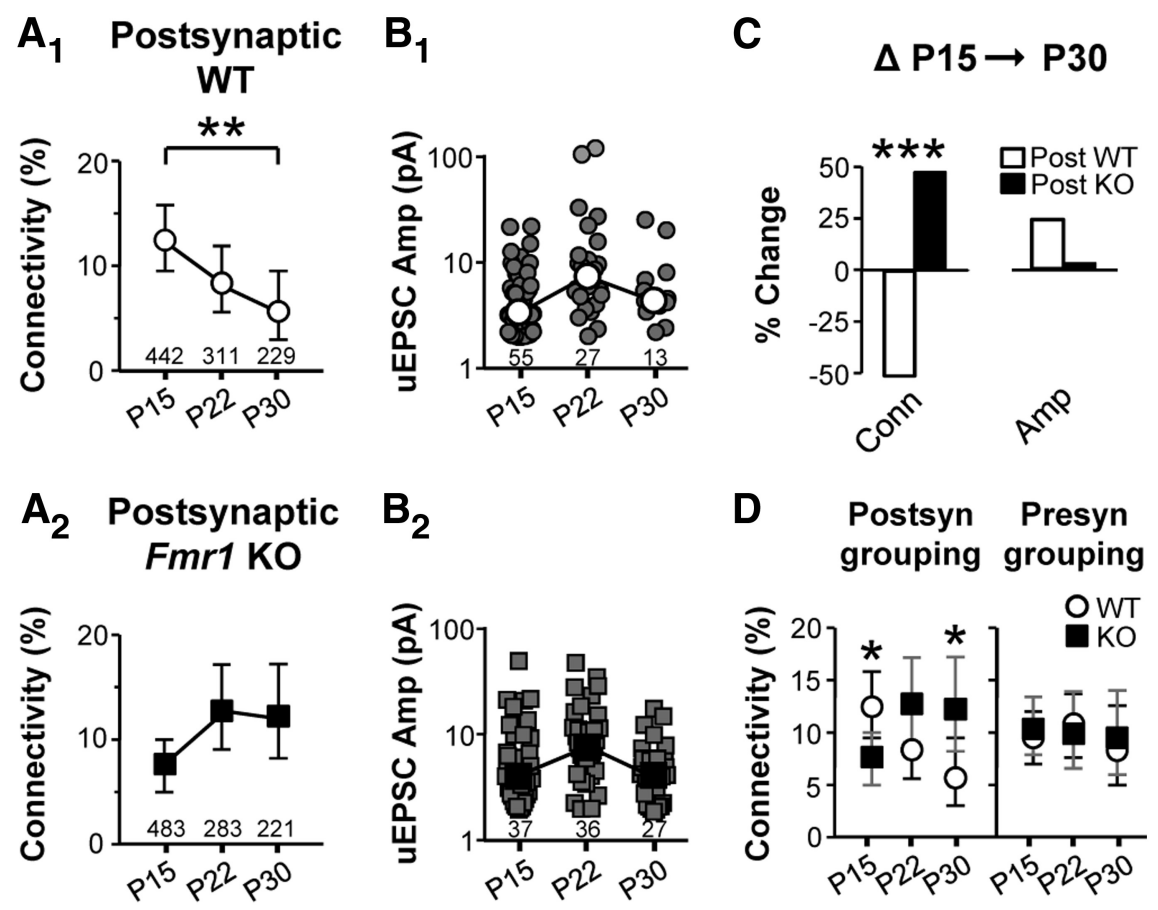

\section{Postsyn Presyn grouping grouping}
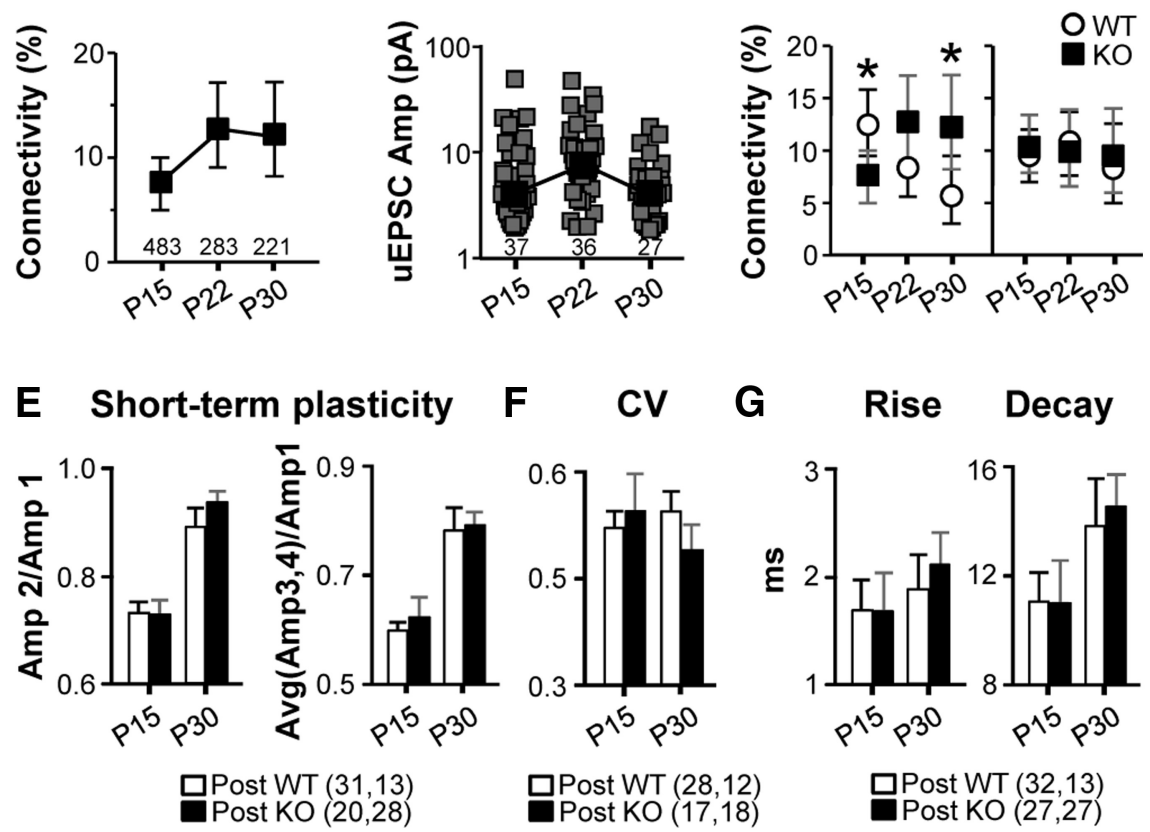

Figure 2. Postsynaptic Fmr 1 regulates the development of cell-to-cell connectivity mediated by AMPA:KA-Rs. $\boldsymbol{A}, \boldsymbol{B}$, Connectivity frequency $(\boldsymbol{A} \mathbf{1}, \boldsymbol{A} \mathbf{2})$, and uEPSC amplitude (B1, B2) are plotted for postsynaptic WT (1) and Fmr $\mathrm{KO}$ (2) cells in mosaic slices. $\boldsymbol{C}$, Percent change in connectivity (Conn) and median amplitude (Amp) in the P15 $\rightarrow$ P30 interval. $\boldsymbol{D}$, Connectivity frequency based on postsynaptic (replot of $\boldsymbol{A}$ ) and presynaptic FMRP expression. $\boldsymbol{E}-\boldsymbol{G}$, Short-term plasticity, $\mathrm{CV}, 20 / 80$ rise-time, and decay time constant of uEPSCs are all unchanged in Fmr $1 \mathrm{~K} 0$ neurons (see Materials and Methods). In $\boldsymbol{E}-\boldsymbol{G}$, numbers in parentheses indicate $n$ (P15, P30).

$\mathrm{Mg}^{2+}$ were applied. Subsequently, uEPSCs mediated by NMDAR were measured at a holding potential of $-50 \mathrm{mV}$. We averaged 50 trials (successes and failures) and a response was considered to exist if the average uEPSC amplitude was $>1.64 \mathrm{pA}$. This value was 3 times the average response of "noise" ( $0.545 \mathrm{pA} ; n=7)$ obtained after blocking a detected NMDARmediated uEPSC with the antagonist ( \pm )-3-(2-carboxypiperazin-4yl)propyl-1-phosphonic acid (CPP; $15 \mu \mathrm{M})$.

Statistics. For continuous data, a Mann-Whitney $t$ test, a KruskalWallis one-way ANOVA on ranks, and two-way ANOVAs were used. ANOVAs were followed by a multicomparisons test. Summary data for uEPSC amplitudes use medians because of their strong non-Gaussian distribution, but an examination of means resulted in similar conclusions. For connectivity frequency, a Pearsons chi-squared test was used and in Figure 1, B, C, F, and Figure 2, $A$ and $D$, a Bonferroni correction based on three testable hypotheses was made. For one-way connections between postnatal day 15 (P15) and P30 in Figure 1C, each presynaptic neuron was a single sample, whereas for two-way connections in the same figure, both presynaptic and postsynaptic neurons were considered a single sample (and not counted again). For Figures $1 E$ and $2 C$, statistical significance for the interaction between age and genotype was used to determine whether the change between P15 and P30 was different between WT and Fmr1 KO. For this, we determined the interaction of $2 \times 2$ proportions (Michael, 2007), followed by a Bonferroni correction based on the 3 possible $2 \times 2$ interactions in our $2 \times 3$ data organization. Measurement error is \pm SEM except for connectivity frequency, which is $95 \%$ confidence interval using the Clopper-Pearson method.

\section{Results}

We first established a time window and the identity of a synaptic pathway that undergoes functionally measured pruning. A previous study made an anecdotal observation that connections among L5A pyramidal neurons might undergo pruning over the first few weeks of postnatal development (Frick et al., 2007). We examined this more closely in L5A of somatosensory cortex. We prepared acute slices from male WT mice at three different ages, P15, $\mathrm{P} 22$, and P30, and performed simultaneous recordings of presynaptic and postsynaptic pyramidal neurons to measure uEPSCs. The uEPSCs were limited to those mediated by AMPA:KA-Rs because of experimental feasibility and because most connections are thought to be mediated by these receptors at P15 and after (Rumpel et al., 2004). We found a monotonic $48 \%$ decrease in connectivity frequency between P15 and P30, which was mainly due to a decrease in "one-way" connections (Fig. 1B1,C1). No change in uEPSC amplitude was observed (Fig. 1D1). These data are consistent with the pruning of connections during normal maturation, when groups of synapses, based on their participation in a single cell-to-cell connection, are pruned together. Moreover, this establishes the $\mathrm{P} 15 \rightarrow \mathrm{P} 30$ time window as a pruning period that most likely includes its beginning because cortical development up to P15 is known to be a period of abundant synaptic and connection formation (Anastasiades and Butt, 2012).

In stark contrast to WT neurons, connectivity frequency in the Fmr1 KO increased by $163 \%$ between P15 and P30. One-way connections did not decrease in frequency and two-way connections increased indicating an aberrant development of connectivity patterns (Fig. 1B2,C2). The connectivity frequency change in the $\mathrm{P} 15 \rightarrow \mathrm{P} 30$ age window was clearly different between WT and the Fmr1 KO (Fig. 1E). Compared with WT, connectivity frequency in the $\mathrm{KO}$ was lower at $\mathrm{P} 15$ but higher at P30 (Fig. $1 F$ ). No change in median uEPSC amplitude was observed for any comparison (Fig. 1D). These data suggest that FMRP promotes connection pruning and its loss results in local hyperconnectivity. The decrease in connectivity at P15 suggests a possible impairment in the net formation of connections.

To determine whether FMRP plays a direct presynaptic or postsynaptic role in regulating connectivity, we performed the same experiment in GFP/Fmr1 mosaic females in which cells 
either coexpress GFP and Fmrl or express neither (Patel et al., 2013). Only when analysis was based on postsynaptic FMRP expression did the results follow the same pattern as the complete WT and Fmr $1 \mathrm{KO}$ (Fig. 2). No changes were detected based on presynaptic groupings. For example, between P15 and P30, the postsynaptic WT connectivity frequency decreased by $52 \%$, whereas no change was detected in the postsynaptic KO group (Fig. 2A1,A2) and hyperconnectivity existed with postsynaptic Fmr1 deletion at P30 (Fig. 2D). The age-dependent increase in connection frequency with postsynaptic Fmr1 deletion was not as striking as that observed in the Fmrl KO, suggesting that non-cell-autonomous factors may enhance the impaired pruning. Unitary EPSC amplitude was not detectably different for any comparison (Fig. 2B). Therefore, postsynaptic FMRP regulates connection frequency and its loss accounts for both the early impoverishment and later increase in connectivity.

In all of the results discussed thus far, uEPSC amplitude was unchanged, suggesting that postsynaptic FMRP only regulates the existence of a connection but not the properties of the connection. This is supported by other unchanged properties of uEPSCs that we examined in mosaic slices at the relevant time points for connectivity alterations (P15 and P30). Short-term plasticity, CV, rise times, and decay time constants of uEPSCs were unchanged with postsynaptic Fmrl deletion at either age (Fig. 2E-G; see Materials and Methods), suggesting that release probability and other connection properties were unchanged. Similarly, no alterations in any of these properties were detected in the constitutive Fmr1 KO (data not shown, all $n=15-30$ ). Short-term plasticity has been demonstrated to increase in the Fmr1 KO due to loss of presynaptic FMRP in the context of long, highfrequency trains (Deng et al., 2013), but not with short trains at the frequency we used. We also observed no change in mEPSC amplitude (see Fig. 4) in mosaic slices, suggesting that the efficacy of single synaptic contacts is unchanged, but we cannot rule out the possibility that a quantal amplitude change is obscured by mEPSCs originating outside of the input pathway we study here. In summary, these data strongly suggest that postsynaptic FMRP does not regulate the properties of a connection when it exists.

Thus far, we have only examined AMPA:KA-R-mediated responses. At the earliest postnatal ages, excitatory synapses are "silent" because they express only NMDARs and not AMPA:KARs. As neocortical circuits mature, silent synapses transition into AMPA:KA-R-containing synapses (Rumpel et al., 2004). In the Fmrl KO, this process is delayed in the thalamocortical pathway

G error bars indicate SEM.
B
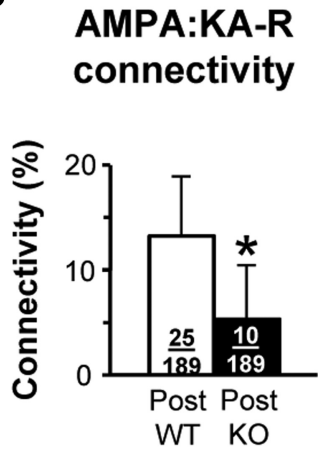

E

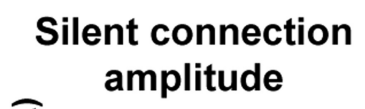

F AMPA:KA/NMDA ratio
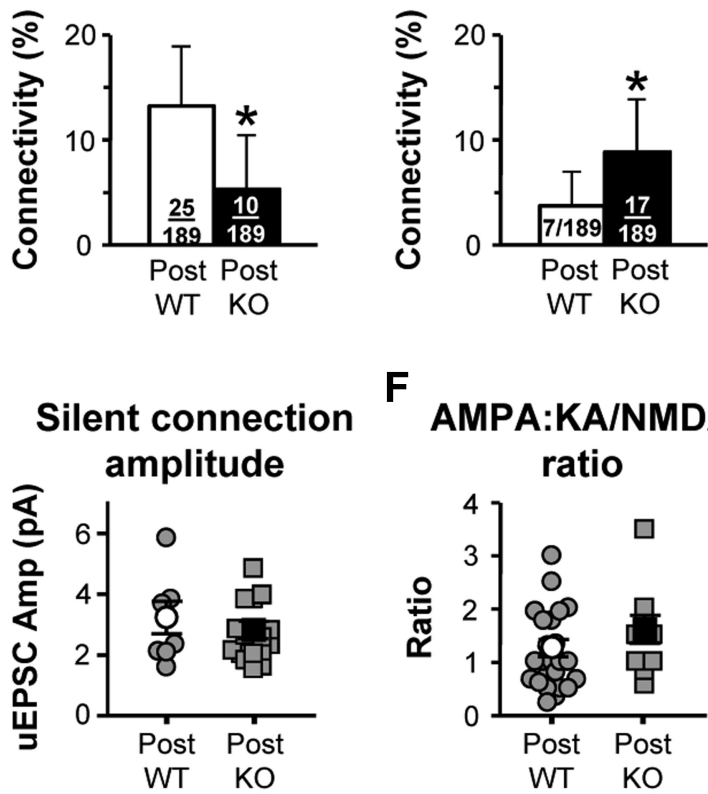

Figure 3. Connectivity frequency is unchanged with postsynaptic Fmr1 deletion at P15 when incorporating an increase in "silent," NMDAR-mediated connections. $\boldsymbol{A}$, Example average uEPSCs that are AMPA:KA-R mediated (top) and silent (bottom). $\boldsymbol{B}, \boldsymbol{C}$

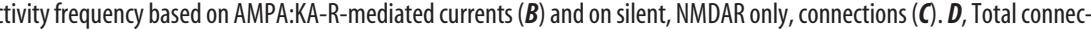
tivity based on adding $\boldsymbol{B}$ and $\boldsymbol{C}$. $\boldsymbol{E}$, uEPSC amplitude of silent connections. $\boldsymbol{F}$, AMPA:KA-R/NMDAR ratio of uEPSCs that were not silent (one WT and two KO uEPSCs had no detectable NMDAR-mediated response and are not included). G, Idealized schematic of our FMRP. This schematic incorporates both silent and AMPA:KA-R-mediated connections and the gray box indicates the estimated time window of exuberant silent connections with Fmr 1 deletion. Error bars in $\boldsymbol{B}-\boldsymbol{D}$ indicate $95 \%$ confidence interval; in $\boldsymbol{E}$ and $\boldsymbol{F}$,

(Harlow et al., 2010). Therefore, connection frequency may be normal at P15 with postsynaptic Fmrl deletion, but with more "silent" connections. We examined both AMPA:KA-R- and NMDAR-mediated responses in P15 (range P14-P15) mosaic slices and found that postsynaptic Fmrl KO neurons had a higher proportion of silent connections (Fig. $3 A-C$ ). Summing the silent and AMPA:KA-R-mediated frequencies (Fig. $3 B, C$ ) into a "total" connectivity frequency, there was no difference between postsynaptic genotypes (Fig. 3D). No effects were observed based on presynaptic FMRP expression. Therefore, FMRP does not regulate connectivity frequency at $\mathrm{P} 15$, but does regulate the maturation of connections. Therefore, there is no excess formation of connections with Fmrl deletion at this early developmental stage. 
A

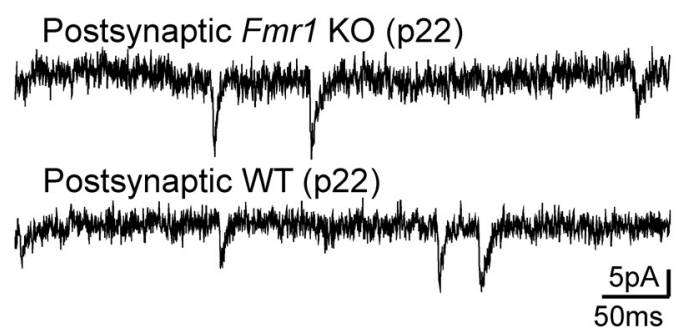

B

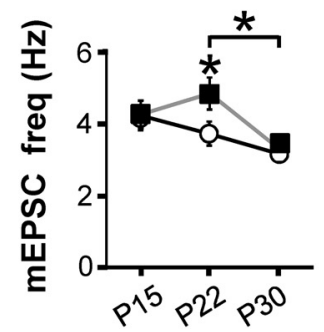

C

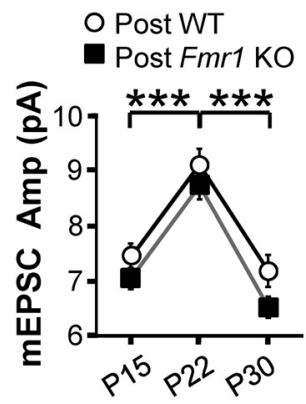

Figure 4. Postsynaptic FMRP's role in connectivity pruning is not observed in mEPSCS. $A$, Example traces of mEPSCs obtained from the mosaic mouse. $B, C$, mEPSC data from mosaic mice did not show either the WT pruning or the pruning deficit with postsynaptic $F m r 1$ deletion in the P15 $\rightarrow$ P30 window. $\mathrm{mEPSC}$ frequency $(\boldsymbol{B})$ and amplitude $(\boldsymbol{C})$ are shown. Although differences are shown for 1 week epochs, none are observed in the context of the P15 $\rightarrow$ P30 interval. Error bars indicate SEM.

If FMRP regulated maturation by synapse and not by cell-tocell connection, one would expect the AMPA:KA-R/NMDAR ratio to be lower in Fmr1 KO neurons that display AMPA:KA-Rmediated responses, but this was not the case (Fig. $3 F$ ). Either connections had a normal AMPA:KA-R/NMDAR ratio or they were completely silent. Therefore, FMRP does not regulate synaptic maturation at the individual synapse level, but in groups based on their participation in a cell-to-cell connection.

Consistent with previous studies documenting the disappearance of silent synapses during cortical development (Rumpel et al., 2004; Harlow et al., 2010; Anastasiades and Butt, 2012), silent connection frequency was practically 0 after $\mathrm{P} 19$ in both postsynaptic WT and KO neurons (WT: 0.9\%, 1/106; KO: 0\%, 0/104; ages P19-P30). This means that the AMPA:KA-R connectivity frequencies at P22 and P30 depicted in Figure 2 accurately represent the "total" connectivity frequency. Therefore, our results indicate that hyperconnectivity with Fmr1 deletion specifically results from impaired "connection" pruning in the later P15 $\rightarrow$ P30 stage (Fig. 3G).

mEPSCs may reflect the total number of synapses from all input pathways onto a neuron. If the pruning we observe is global on the neuron, we might expect a decrease in mEPSC frequency in the P15 $\rightarrow$ P30 interval in WT neurons but not in Fmr1 KO neurons. We examined mEPSCs in WT and Fmr1 KO neurons of mosaic slices. Although we did observe a change in mEPSC frequency at P22 in the Fmr1 KO (Fig. 4B), we observed no changes at the ages relevant to our evoked transmission results, the P15 and P30 time points. There was no detectable change in mEPSC frequency between P15 and P30 for either WT or KO neurons (Fig. 4B). Neither were there any differences between WT and KO neurons at these ages. These data suggest that pruning may be largely restricted to local L5A input and may not be a global input process, but caution should also be applied to this interpretation because mEPSCs may be regulated independently of evoked transmission (Ramirez and Kavalali, 2011). As with mEPSC frequency, there was no change at these time points under any comparison for mEPSC amplitude.

Postsynaptic membrane properties likely did not significantly affect our results because we observed no changes in input resistance at $-60 \mathrm{mV}$ due to Fmr1 deletion in the mosaic mice at any age (in $\mathrm{M} \Omega$ : $\mathrm{P} 15,348 \pm 24$ vs $336 \pm 18, n=37,55 ; \mathrm{P} 22,223 \pm 11$ vs $225 \pm 15, n=25,22$; P30, $233 \pm 10$ vs $229 \pm 8, n=81,76$ for $\mathrm{WT}$ vs $\mathrm{KO}$, respectively). Interestingly, input resistance was higher at P15 and P30 in Fmr1 KO mice (in M $\Omega$ : P15, $277 \pm 14$ vs $454 \pm 23 \mathrm{M} \Omega, p<0.01, n=42,51 ; \mathrm{P} 30,190 \pm 7$ vs $249 \pm 12 \mathrm{M} \Omega$, $p<0.01, n=41,53$ for WT and KO, respectively), suggesting that regulation of some membrane properties by FMRP is not cell autonomous.

\section{Discussion}

We demonstrate here that local interconnections of L5A pyramidal neurons undergo "connection pruning" between the third and fifth postnatal weeks. With the establishment of this pruning model system, we were able to show that pruning is impaired in the Fmr1 KO mouse due to loss of postsynaptic FMRP. We find that hyperconnectivity in the L5A network in the Fmr1 KO stems from this pruning impairment. We also observe a role for FMRP in synapse maturation. In both its pruning and maturation functions, FMRP regulates cell-to-cell connections in which groups of synapses are pruned, retained, or matured together based on their involvement in a single cell-to-cell connection. We also make the first observations of changes in the development of connection patterns (i.e., one-way versus two-way connections) in a mouse model of intellectual disability.

The pruning process that we observe may be layer, region, and cell-type specific. Unlike the hyperconnectivity we observe in L5A at P30, connectivity among layer 4 excitatory neurons in the same cortical region at a comparable age is most likely unchanged or slightly decreased in the Fmr1 KO (Gibson et al., 2008). Hyperconnectivity among L5 neurons in prefrontal cortex of the Fmr1 KO mouse is only observed during the third postnatal week and, by the fifth week, connectivity is normal (Testa-Silva et al., 2012). In this latter study, it is not clear whether the L5 neurons were intratelencephalic (IT) or pyramidal tract projecting neurons, the two general subtypes of neocortical pyramidal neurons (Shepherd, 2013). The L5A neurons in our study were the IT subtype. Therefore, it is possible that loss of FMRP most strongly affects pruning among IT neurons.

We cannot rule out the possibility that pruning eventually does occur in L5A in the Fmr1 KO and that it is dramatically delayed to later ages. Even so, our results indicate that, over a significant time period of L5A circuit development (at least 2 weeks), connectivity is abnormal, which could greatly affect learning and circuit development. Hyperconnectivity through excess feedback excitation could underlie the circuit hyperexcitability in somatosensory cortex observed in the first 4 postnatal weeks in the Fmr1 KO (Hays et al., 2011; Gonçalves et al., 2013).

The increased prevalence of "silent" synapses with Fmr1 deletion has been reported previously in the thalamocortical pathway into somatosensory cortex (Harlow et al., 2010). We build on this earlier finding in two ways. First, FMRP promotes the maturation of synapses in multiple cortical synaptic pathways. Second, and more significantly, FMRP promotes maturation at the level of cell-to-cell connections. A similar role for postsynaptic FMRP in promoting synapse maturation has been reported in hippocampal cultured slices (Zang et al., 2013), in which the maturation 
was not in the context of silent synapses, but in the transition of NMDAR subtypes. Interestingly, our results suggest that the silent connections at P15 in Fmr1 KO neurons may either be transformed into or replaced by AMPA:KA-mediated connections that fail to undergo normal pruning, but resolving this would require future study.

\section{References}

Anastasiades PG, Butt SJ (2012) A role for silent synapses in the development of the pathway from layer $2 / 3$ to 5 pyramidal cells in the neocortex. J Neurosci 32:13085-13099. CrossRef Medline

Bagni C, Greenough WT (2005) From mRNP trafficking to spine dysmorphogenesis: the roots of fragile X syndrome. Nat Rev Neurosci 6:376-387. CrossRef Medline

Bakker CE, Verheij C, Willemsen R, van der Helm R, Oerlemans F, Vermey M, Bygrave A, Hoogeveen AT, Oostra BA, Reynierse E, De Boulee K, D’Hoogeg R, Crasf P, van Velzene D, Nagelsg G, Martin J-J, De Deyng PP, Darbye JK, Willems PJ; The Dutch-Belgian Fragile X Consortium (1994) Fmrl knockout mice: a model to study fragile X mental retardation. Cell 78:23-33. CrossRef Medline

Bureau I, Shepherd GM, Svoboda K (2008) Circuit and plasticity defects in the developing somatosensory cortex of FMR1 knock-out mice. J Neurosci 28:5178-5188. CrossRef Medline

Contractor A, Mulle C, Swanson GT (2011) Kainate receptors coming of age: milestones of two decades of research. Trends Neurosci 34:154-163. CrossRef Medline

Deng PY, Rotman Z, Blundon JA, Cho Y, Cui J, Cavalli V, Zakharenko SS, Klyachko VA (2013) FMRP regulates neurotransmitter release and synaptic information transmission by modulating action potential duration via BK channels. Neuron 77:696-711. CrossRef Medline

Frick A, Feldmeyer D, Sakmann B (2007) Postnatal development of synaptic transmission in local networks of L5A pyramidal neurons in rat somatosensory cortex. J Physiol 585:103-116. CrossRef Medline

Gibson JR, Bartley AF, Hays SA, Huber KM (2008) Imbalance of neocortical excitation and inhibition and altered UP states reflect network hyperexcitability in the mouse model of fragile X syndrome. J Neurophysiol 100: 2615-2626. CrossRef Medline

Gonçalves JT, Anstey JE, Golshani P, Portera-Cailliau C (2013) Circuit level defects in the developing neocortex of Fragile X mice. Nat Neurosci 16: 903-909. CrossRef Medline

Hadjantonakis AK, Cox LL, Tam PP, Nagy A (2001) An X-linked GFP transgene reveals unexpected paternal X-chromosome activity in trophoblastic giant cells of the mouse placenta. Genesis 29:133-140. CrossRef Medline

Hanson JE, Madison DV (2007) Presynaptic FMR1 genotype influences the degree of synaptic connectivity in a mosaic mouse model of fragile $\mathrm{X}$ syndrome. J Neurosci 27:4014-4018. CrossRef Medline

Harlow EG, Till SM, Russell TA, Wijetunge LS, Kind P, Contractor A (2010) Critical period plasticity is disrupted in the barrel cortex of FMR1 knockout mice. Neuron 65:385-398. CrossRef Medline

Hays SA, Huber KM, Gibson JR (2011) Altered neocortical rhythmic activity states in Fmr1 KO mice are due to enhanced mGluR5 signaling and involve changes in excitatory circuitry. J Neurosci 31:14223-14234. CrossRef Medline

Holtmaat A, Svoboda K (2009) Experience-dependent structural synaptic plasticity in the mammalian brain. Nat Rev Neurosci 10:647-658. CrossRef Medline

Kano M, Hashimoto K (2009) Synapse elimination in the central nervous system. Curr Opin Neurobiol 19:154-161. CrossRef Medline

Michael GA (2007) A significance test of interaction in $2 \times \mathrm{K}$ designs with proportions. Tutor Quant Meth Psych 3:1-7.

Patel AB, Hays SA, Bureau I, Huber KM, Gibson JR (2013) A target cellspecific role for presynaptic Fmr1 in regulating glutamate release onto neocortical fast-spiking inhibitory neurons. J Neurosci 33:2593-2604. CrossRef Medline

Pfeiffer BE, Huber KM (2007) Fragile X mental retardation protein induces synapse loss through acute postsynaptic translational regulation. J Neurosci 27:3120-3130. CrossRef Medline

Rakic P, Bourgeois JP, Eckenhoff MF, Zecevic N, Goldman-Rakic PS (1986) Concurrent overproduction of synapses in diverse regions of the primate cerebral cortex. Science 232:232-235. CrossRef Medline

Ramirez DM, Kavalali ET (2011) Differential regulation of spontaneous and evoked neurotransmitter release at central synapses. Curr Opin Neurobiol 21:275-282. CrossRef Medline

Rumpel S, Kattenstroth G, Gottmann K (2004) Silent synapses in the immature visual cortex: layer-specific developmental regulation. J Neurophysiol 91:1097-1101. Medline

Shepherd GM (2013) Corticostriatal connectivity and its role in disease. Nat Rev Neurosci 14:278-291. CrossRef Medline

Tessier CR, Broadie K (2008) Drosophila fragile X mental retardation protein developmentally regulates activity-dependent axon pruning. Development 135:1547-1557. CrossRef Medline

Testa-Silva G, Loebel A, Giugliano M, de Kock CP, Mansvelder HD, Meredith RM (2012) Hyperconnectivity and slow synapses during early development of medial prefrontal cortex in a mouse model for mental retardation and autism. Cereb Cortex 22:1333-1342. CrossRef Medline

Zang T, Maksimova MA, Cowan CW, Bassel-Duby R, Olson EN, Huber KM (2013) Postsynaptic FMRP bidirectionally regulates excitatory synapses as a function of developmental age and MEF2 activity. Mol Cell Neurosci 56:39-49. CrossRef Medline 